\title{
Non-central Serous Chorioretinopathy
}

\author{
Relli Ovadia Bronshtein, Keren Chet-Shoer, Shimon Rumelt \\ Department of Ophthalmology, Western Galilee-Nahariya Medical Center, Nahariya, Israel
}

\begin{abstract}
Central serous chorioretinopathy occupies the macula and has been described in association with different conditions, including pregnancy. In this report, a 23-year-old pregnant woman underwent uneventful caesarean section in the 40th week of pregnancy after failure of vaginal delivery. One day after the delivery, the patient complained of blurred vision in her left eye and was found to have atypical, eccentric serous chorioretinopathy that subsequently resolved gradually. Serous chorioretinopathy may be atypical in location and occur after delivery because of high blood pressure, hormonal changes, or stressogenic delivery.
\end{abstract}

Key words: Central serous chorioretinopathy, Cesarean section, Choroid diseases, Etiology, Pregnancy, Retinal diseases

Asian J Ophthalmol. 2011;12:175-6.

\section{Introduction}

Central serous chorioretinopathy (CSCR) is a neurosensory retinal detachment occupying the central macula and causing decrease in visual acuity. ${ }^{1,2}$ The condition affects young individuals, many of whom have a history of a recent stressogenic event or type A personality, and has been found to be associated with systemic corticosteroid, antibiotic or alcohol use, pregnancy, uncontrolled hypertension, and allergic respiratory distress. ${ }^{2}$

This report is of a patient who developed serous chorioretinopathy located outside the macula (non-central serous chorioretinopathy [NCSCR]) following childbirth, a situation that, to the authors' knowledge, has not previously been reported in the literature.

\section{Case Report}

A 23-year-old pregnant woman (gravida 1, para 0) was admitted to the Western Galilee Hospital, Nahariya, Israel, in 2009, in the 40th week of pregnancy with uterine contractions. Her blood pressure at admission was 148/101 mm Hg, and she was diagnosed with preeclampsia. A vaginal delivery failed, even when intravenous oxytocin $1 \mathrm{mU} /$ minute was started and the dose increased gradually. The patient underwent uneventful Caesarean section and was treated afterwards with oral nifedipine $30 \mathrm{mg} /$ day. One day after delivery, the patient had blurred vision in her left eye. Ocular examination showed that her best-corrected visual acuity was 20/20 in the right eye and 20/60 in the left eye. Her intraocular pressure and anterior

Correspondence: Dr Shimon Rumelt, Department of Ophthalmology, Western Galilee-Nahariya Medical Center, PO Box 21, 22100 Nahariya, Israel.

Tel: (972 4) 910 7635;

E-mail: shimon.rumelt@naharia.health.go.il segment were normal. A vesicle-like elevation of the neurosensory retina was found over the inferior temporal arcade (Figure 1). Optical coherence tomography demonstrated elevation of the neurosensory retina corresponding to the vesicle-like elevation (Figure 2). The patient was followed for 3 months until resolution of the retinal lesion and visual acuity improved to 20/30. The patient underwent fluorescein angiography after resolution of the finding and the result was normal.

\section{Discussion}

A unique feature of SCR in this patient was its non-central location. To the authors' knowledge, this is the first report of such a locality.

Figure 1. Atypical non-central location of serous chorioretinopathy (arrows) located at the inferior temporal arcade in a 23-year-old woman after delivery.

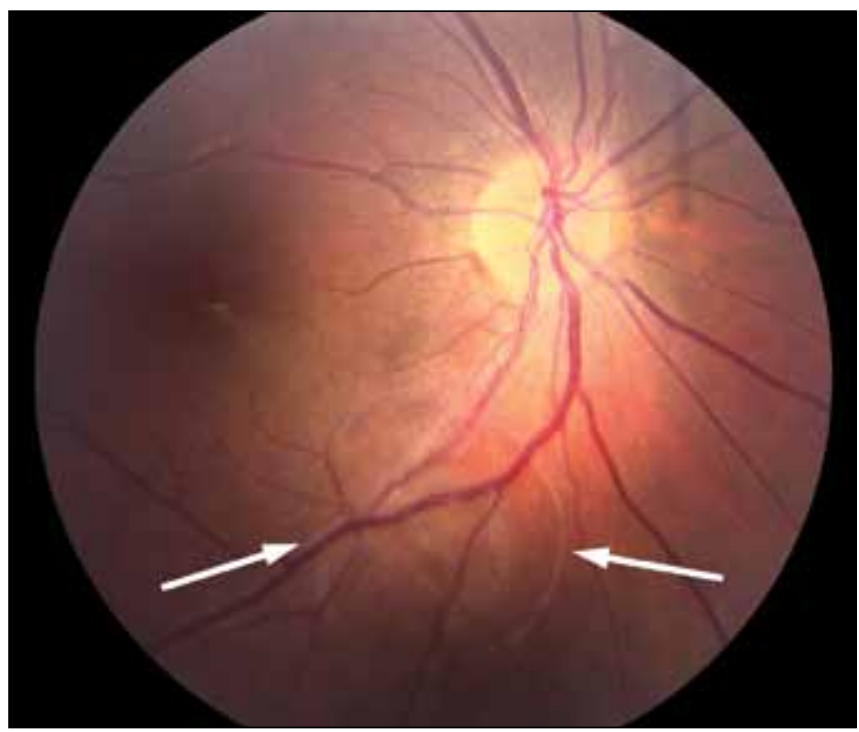


Figure 2. Optical coherence tomography showing separation of the neurosensory retina at the inferior temporal arcade.

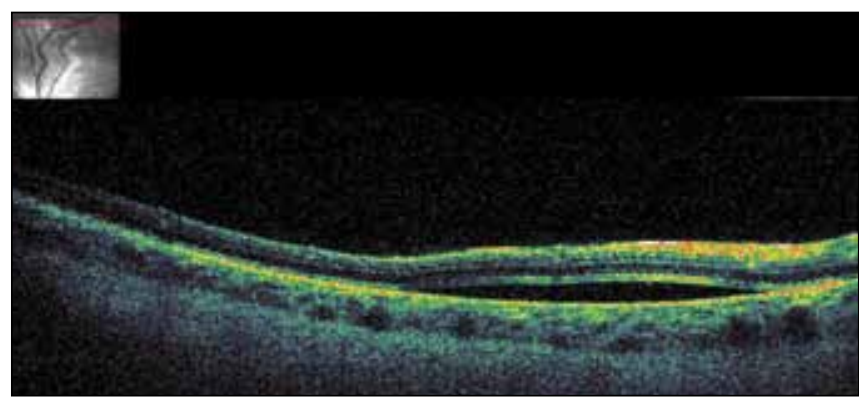

There might be similar cases with an atypical eccentric location that did not cause any symptoms or that were overlooked because of the location. Therefore, it is essential to meticulously examine the entire retina in patients who have decreased or blurred vision. The decrease in vision might have been caused by extension of the CSCR to the fovea when the patient became symptomatic. Since the lesion is usually self-limiting, if it was present before delivery, it was probably only for a limited time.

Although CSCR has been associated with pregnancy, ${ }^{2}$ it has not been described to occur immediately following delivery, according to a literature search of Medline and Index Medicus. Several possible causative factors may have played a role in the development of serous chorioretinopathy in this patient, including high blood pressure at admission, hormonal changes, and a stressogenic delivery. Administration of oxytocin to induce labour may also be a contributing cause, although this has never been described. The differential diagnosis of CSCR includes exudative retinal detachment and acute macular retinopathy, but these conditions have different clinical appearance and course. The normal fluorescein angiography after the resolution of the finding further supports the diagnosis of CSCR.

After delivery, women should be examined for CSCR if they complain of decreased vision and the location of the CSCR might be atypical.

\section{References}

1. Sunness JS. The pregnant woman's eye. Surv Ophthalmol. 1988;32 219-38.

2. Haimovici R, Koh S, Gagnon DR, Lehrfeld T, Wellik S; Central Serous Chorioretinopathy Case-Control Study Group. Risk factors for central serous chorioretinopathy: a case-control study. Ophthalmology. 2004;111:244-9. 